\title{
SUR UN PIROPLASME ATYPIQUE \\ DE DEUX BOVIDÉS DU CONGO
}

\section{Par J. SCHWETZ}

Dans une note précédente (Schwetz et Storck, 1930) sur un cas de triple infection d'une vache par Piroplasma bigeminum, Gonderia mutans et Trypanosoma uniforme, nous disions :

1. Que la trypanosomose des bovidés était très commune à Stanleyville ;

2. Que les infections à Gonderia mutans n’y étaient pas rares non plus ;

3. Que la piroplasmose (ou babésiose), sensu stricto, à Piroplasma bigeminum y était par contre extrêmement rare, si rare que nous n'en avons trouvé qu'un seul cas après deux années et demie de recherches et après avoir examiné environ un millier de bovidés. C'était d'ailleurs une des raisons pour lesquelles nous avions cru utile de signaler ce cas. Il s'agissait d'une vache de Stanleyville.

Sur près de mille bovidés examinés dans diverses localités de l'Uélé, nous avons également trouvé de nombreux cas de trypanosomose et quelques rares cas de Gonderia mutans, mais pas de Piroplasma sensu stricto: ni P. bigeminum, ni une autre forme.

Peu de temps après avoir envoyé la note précédente, nous eûmes l'occasion de constater deux cas d'une piroplasmose un peu spéciale que nous hésitons à déterminer et que nous croyons intéressant de signaler.

Il s'agit d'un petit troupeau de bovidés amené en quelques jours (en auto-camion) de Dili (Uélé) à Stanleyville. Ce troupeau avait été trouvé précédemment (sur place, à Dili), fortement infecté par la trypanosomose. Nous envoyons les quelques bêtes trouvées indemnes dans une ferme voisine et gardons au laboratoire les autres, parasitées par Trypanosoma congolense et $T$. vivax. En outre, nous gardons deux bêtes indemnes pour la raison suivante : une de ces bêtes est la mère d'un veau trypanosomé et l'autre est un veau indemne, né d'une mère trypanosomée. Chose bizarre, ce sont justement ces deux bêtes indemnes qui meurent presque subitement de piroplasmose.

Annales de Parasitologie, $\mathrm{T}$. VIII, $\mathrm{N}^{\circ} 6 .-1^{\text {er }}$ décembre 1930, p. 602-609. 
Première bête (fig. 1). - Vache de Dili, $N^{\circ} 77$. Meurt presque subitement dans la nuit du 23 au 24 décembre. Le 25 au matin, examen du sang du cœur, en goutte épaisse et en frottis. Pas de trypanosomes, mais déjà, dans la goutte épaisse, on voit des hématozoaires rappelant un peu les schizontes des parasites du paludisme.

Dans les frottis, on constate avant tout une forte anisocytose, mais il ne faut pas oublier qu'il s'agissait de sang prélevé post mortem. Nombreux globules polychromatrophiles. Corps de Jolly et même normoblastes. Piroplasmes assez nombreux (voir plus loin), de formes les plus variées. Quant à la taille des parasites, elle est également très variable. Certains parasites ne sont pas plus grands que ceux des theilérioses; d'autres sont presque aussi grands que Piroplasma bigeminum ; mais la plupart ont des dimensions moyennes: plus grandes que Theileria-Gonderia, msis plus petites que $P$. bigeminum. Bref, les dimensions des parasites (par rapport aux hématies) font penser aux parasites d'une babésiellose.

Comme nous l'avons déjà dit, la vache est morte presque subitement. L'autopsie n'a rien révélé d'anormal.

Deuxième bête (fig. 2). - Veau du même troupeau, âgé de 4 à 5 mois.

Tombe malade le 29 décembre. T. $38^{\circ} 5$. P. 110 . R. 12 . Conjonctives blanc sale. Sang très aqueux. Examen microscopique; pas de trypanosomes, mais très rares Gonderia mutans, formes bacillaires.

Le lendemain, 30 décembre, le veau est presque rétabli. T. $37^{\circ} 5$. P. 80. R. 10. Sang toujours aqueux. Pas de trypanosomes à l'examen à l'état frais. Pas d'autres examens.

Le jour suivant, 31 décembre, le veau se couche et meurt avant que l'on ait eu le temps de l'examiner. Les frottis de sang du cœur, prélevé immédiatement après la mort, donnent à peu près le même tableau que ceux de la vache : anisocytose, polychromatophilie et piroplasmes de formes et de dimensions variées, toutefois beaucoup plus rares que chez la vache. L'autopsie n'a rien révélé d'anormal : ni hémoglobinurie, ni ictère. Nous n'avons pas trouvé de corpuscules de Kòch dans les ganglions lymphatiques ni dans la rate.

\section{Discussion}

De quelle espèce de piroplasmose s'agit-il dans nos deux cas ? Les renseignements cliniques nous manquent. Les constatations 


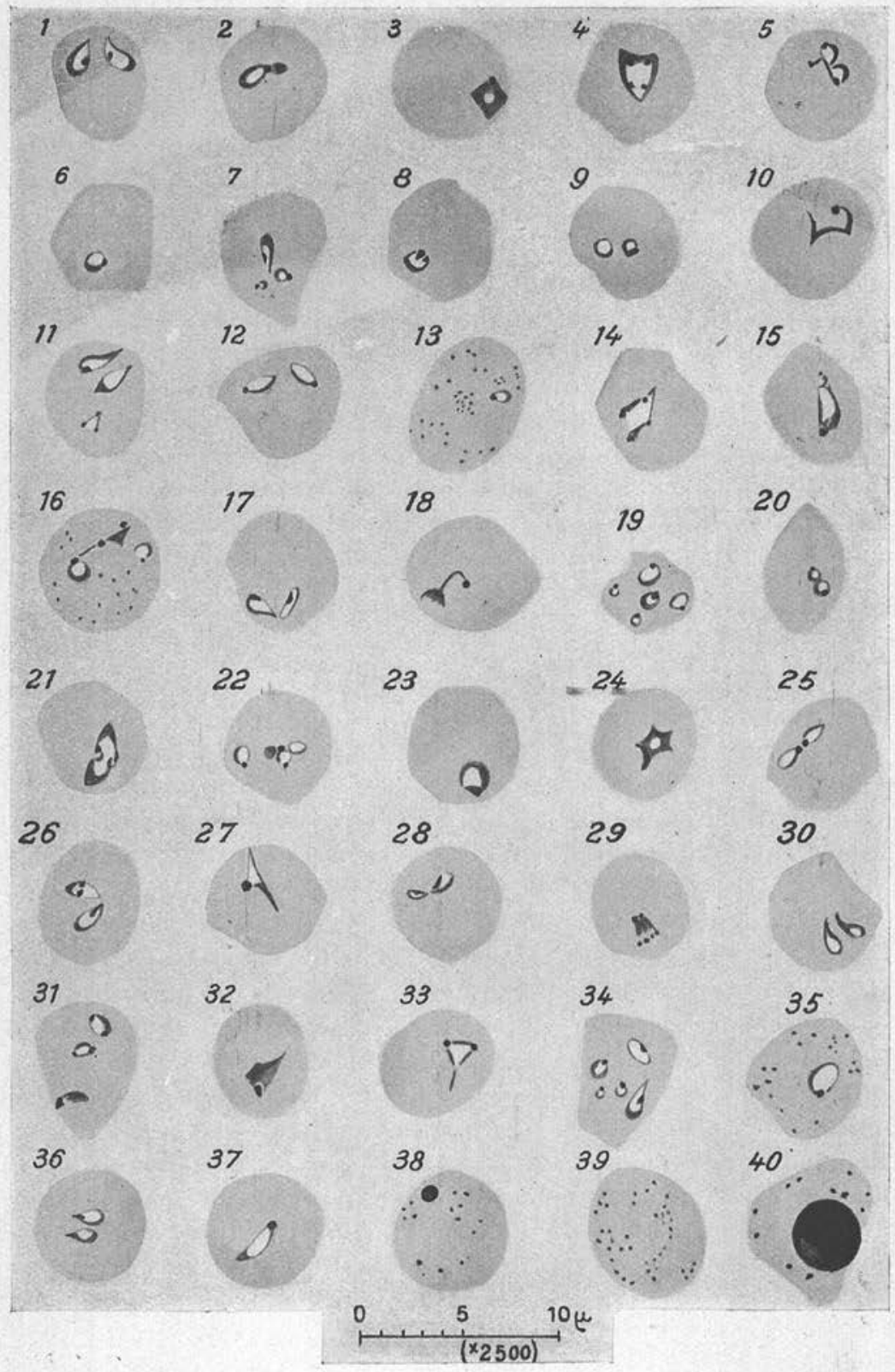

Fig. 1. - Piroplasma sp. d'une vache. 
anatomo-pathologiques sont nulles. Les tiques trouvées par nous sur les animaux des divers troupeaux sont des espèces africaines communes: Amblyomma variegatum, Boophilus (Margaropus) decoloratus et Rhipicephalus appendiculatus. On sait que les deux dernières espèces sont incriminées dans la transmission de Piro-

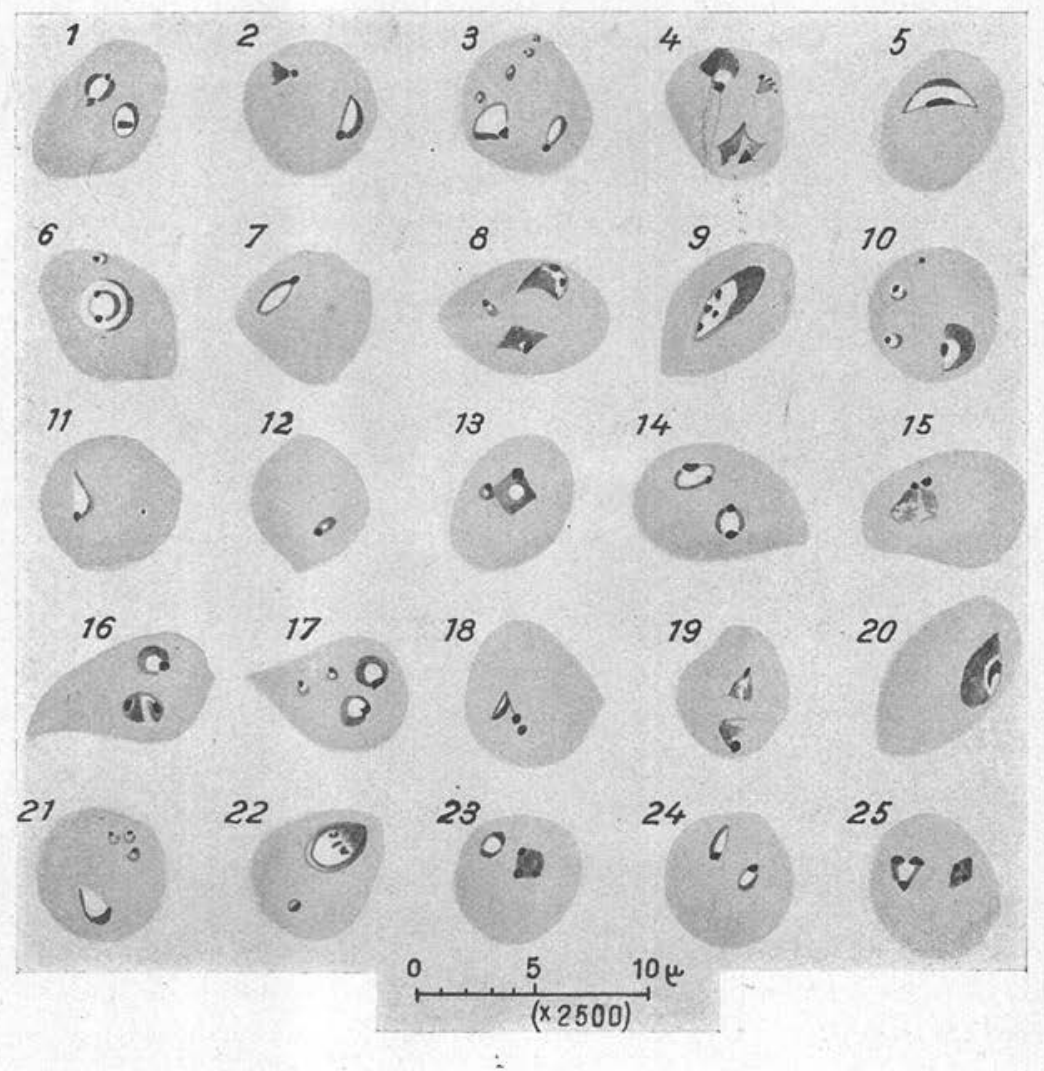

Fig. 2. - Piroplasma sp. d'un veau.

plasma bigeminum et la dernière, de Gonderia mutans. Mais il est évident que la transmission d'une autre espèce de Piroplasma par ces tiques n'est pas exclue. De sorte que le seul moyen que nous possédions pour déterminer notre piroplasmose, c'est sa morphologie.

On distingue les diverses espèces de piroplasmose des bovidés suivant la taille et la forme des parasites, et, en partie, suivant la proportion des globules parasités. 
1. Piroplasma bigeminum est caractérisé par des éléments piriformes de grande taille, atteignant le diamètre du globule.

2. Piroplasma (ou Babesiella) bovis est caractérisé par des éléments piriformes de petite taille, formant souvent un angle assez grand $(P$. divergens) et situées à la surface du globule.

3. Piroplasma argentinum ressemble morphologiquement à $P$. bovis, avec cette différence que les parasites sont très rares et les éléments bigéminés divergents ne se trouvent pas à la surface du globule.

Nous nous bornons à indiquer ici les principales formes caractéristiques pour chacune des trois espèces énumérées. Parce que, en réalité, la question est beaucoup plus compliquée, chacune de ces espèces possédant encore d'autres formes de transition et partant moins typiques et plus ou moins communes à toutes les espèces, notamment des formes rondes et irrégulières.

C'est ainsi que Piroplasma (Babesiella) bovis est caractérisé par la prédominance des formes rondes sur les formes ovalaires. Mais, d'autre part, la description de Piroplasma bovis, donnée par Brumpt, est différente de celle de Babesiella donnée par Sergent et ses collaborateurs.

4. Gonderia mutans. - Nous ne nous arrêterons pas sur cette espèce qui est caractérisée par de très petits parasites ressemblant aux divers Theileria. Mais Gonderia mutans est souvent associé aux piroplasmoses proprement dites. Et s'il n'est pas difficile de déterminer une infection pure à Gonderia mutans, ce parasite complique parfois la détermination quand il est associé à un autre piroplasme comme dans nos deux cas.

Quoique les parasites trouvés chez la vache et chez le veau se ressemblent, chacune des deux infections présente certaines particularités. Si on compare les deux figures, on constate que dans les deux cas il s'agit d'une association de deux espèces de parasites.

Gonderia mutans existe certainement dans les deux cas. Par exemple fig. 1 (vache), $\mathrm{n}^{\circ} 11$ et fig. 2 (veau), $\mathrm{n}^{\circ \mathrm{s}} 3$ et 21 et probablement $6,10,17$ et 22 .

Il est plus difficile de trancher le caractère de plusieurs autres petits parasites, par exemple dans les hématies $9,16,19$ et 34 de la fig. 1 et dans les $n^{\circ s} 8,10,12$ et 17 de la fig. 2 . Si nous avions affaire à une infection pure à Gonderia mutans, nous n'aurions pas hésité à rattacher ce parasite un peu douteux à la même espèce. Mais puisqu'il s'agit encore d'une infection simultanée d'un vrai piroplasme de formes et de tailles variées, nous nous réservóns. 
A ce propos, nous croyons utile d'attirer l'attention sur certaines particularités de coloration que nous n'avons pas trouvées signalées chez les auteurs. Avec les colorations habituelles, le cytoplasme de tous les piroplasmes est coloré en bleu et la chromatine en rouge. Mais il y a des nuances sous ce rapport entre les piroplasmes proprement dits, d'une part, et Gonderia mutans, d'autre part. Le protoplasme de ce dernier est plus clair (bleu céleste) et la chromatine l'est également (rouge géranium). Les contours de la petite masse chromatique sont en outre bien définis et nets. Tandis que dans les piroplasmes, le bleu du protoplasme est plus foncé (bleu pastel) et la chromatine est d'un rouge également plus foncé, plus brunâtre (rouge cramoisi). Les contours de la masse chromatique sont en outre moins nets. Mais ce n'est que dans les préparations très bien colorées qu'on peut observer ces nuances qui ne sont évidemment que relatives.

Outre l'infection à Gonderia mutans, nous avions affaire dans nos deux cas à une autre infection à vrais piroplasmes. Mais si déjà à l'examen des frottis nous nous demandions à quelle espèce de piroplasme nous avions affaire, la reproduction des divers parasites a encore augmenté notre perplexité.

Voici d'ailleurs quelques renseignements statistiques :

\section{VAche (fig. 1)}

A. Sur 7.000 érythrocytes comptés, nous en avons trouvé 616 parasités $(8,90 / 0)$, dont 28 par des Gonderia (Theileria) mutans $(0,40 / 0)$ et 588 par des piroplasmes proprement dits $(8,50 / 0)$.

B. Sur ces 7.000 globules rouges, nous avons, en outre, trouvé : 7 normoblastes, 4 avec des corps de Jolly et 171 polychromatophiles.

C. Sur les 588 globules parasités par des piroplasmes, nous avons dénombré les formes suivantes :

1. 141 poires simples.

2. 161 poires doubles (libres).

3. 1 poire quadruple (libre).

4. 1 poire quintuple (libre).

5. 17 poires bigéminées (liées par un pédicule) à angle aigu.

6. 1 poire bigéminée divergente et périphérique.

7. 150 formes rondes simples.

8. 64 formes rondes doubles.

$9 . \quad 2$ formes rondes triples.

10. 30 formes irrégulières simples.

11. 20 formes irrégulières multiples. 


\section{VEAU (fig. 2)}

A. Sur 7.000 érythrocytes comptés nous avons trouvé 126 globules parasités $(1,80 / 0)$ dont 62 par de petits parasites, Gonderia (Theileria) mutans $(0,90 / 0)$, et 64 par des piroplasmes proprement dits $(0,90 / 0)$.

B. Sur les 64 piroplasmes, nous avons dénombré les formes suivantes :

1. 7 poires simples.

2. 3 poires doubles (deux par globules).

3. 5 formes rondes simples.

4. 12 formes rondes doubles (deux par globules).

5. 11 formes irrégulières simples.

6. 26 formes irrégulières doubles (deux par globules).

Il résulte de cette statistique que les Gonderia (Theileria) mutans sont plus nombreux chez le veau $(0,90 / 0)$ que chez la vache $(0,40 / 0)$. En ce qui concerne les piroplasmes proprement dits, qui font le sujet de cette note, c'est juste le contraire. Les globules parasités sont très rares chez le veau $(0,90 / 0)$ et relativement assez nombreux chez la vache $(8,50 / 0)$.

Quant à la morphologie proprement dite, il serait trop long de décrire et même d'énumérer toutes les formes que nous avons vues. Il était même difficile de les reproduire toutes dans les figures.

On peut dire que les formes trouvées chez la vache et chez le veau se ressemblent. Proportionnellement, il y a beaucoup de formes rondes ou légèrement ovalaires de 1 à $2 \mu$. Les éléments piriformes ont en moyenne $2 \mu$ de longueur, parfois un peu plus. Quoique petites, les quelques poires bigéminées trouvées dans le sang de la vache sont convergentes, à angle aigu. Nous avons toutefois reproduit une exception dans le $n^{\circ} 28$ de la fig. 1 : une forme bigéminée divergente. Une autre forme analogue, trouvée une seule fois, une forme bigéminée périphérique n’a pas été dessinée.

Quant aux formes irrégulières, elles sont si variées, aussi bien par la taille que par la forme, que nous nous sommes bornés à faire dessiner quelques-unes d'entre elles. Il y en a qui ressemblent à un écusson (4, fig. 1), à une harpe (29), ou à une étoile (14). La taille des formes irrégulières atteint quelquefois 3 et même $4 \mu$. C'est dans le sang du veau que nous avons trouvé les formes les plus grandes et notamment une forme elliptique atteignant $5 \mu(9$, fig. 2$)$.

Aussi bien dans la vache que dans le veau, il s'agit évidemment d'une infection mixte : à Gonderia (Theileria) mutans, d'abord, et à Piroplasma sensu stricto, ensuite. Nous n’avons pas à nous arrêter ici sur l'infection par Gonderia (Theileria) mutans, qui ne pré- 
sente rien de particulier dans nos deux cas. Mais quel est notre piroplasme ? Sa taille est plus petite que la taille habituelle de Piroplasma bigeminum, et, par contre, un peu plus grande que celle de Piroplasma (Babesiella) bovis. Il en est de même de la forme de nos parasites, qui est aussi atypique pour $P$. bigeminum que pour $P$. bovis. S'agit-il d'une infection mixte par ces deux espèces, d'une infection double (et même triple, en comptant Gonderia mutans) ? ou d'une infection à Piroplasma bigeminum atypique ? ou, enfin, d'une espèce spéciale de Piroplasma? C'est ce que nous laissons à d'autres le soin de juger et de trancher.

En terminant notre note, il nous reste à remercier notre collaborateur, le vétérinaire Storck, et notre assistante, Mlle Droeshaut : le premier pour la partie préliminaire et routinière de notre travail et la seconde pour l'exécution des deux figures jointes à cette note.

\section{BIBLIOGRAPHIE}

Brumpt (E.). - Les piroplasmoses des bovidés et leurs hôtes vecteurs. Bull. Soc. pathol. exot., XIII, 1920, p. 416-460.

Kinuth (P.) et De Tort (P.-J.). - Tropenkrankheiten der Haustiere, in Mense, Handbuch der Tropenkrankheiten, VI, 1921, Leipzig.

Mesnil (F.). - Piroplasmes. Cours de microbiologie de l'Institut Pasteur, 1924 (inédit).

Schwetz (J.) et Storck (N.), - On a case of triple infection in a cow : Piroplasma bigeminum, Gonderia mutans et Trypanosoma uniforme. Trans. Roy. Soc. trop. med, and hyg., XXIII, 1930, p. 637-639.

Sergent (Ed.), Donatien (A.), Parrot (L.), Lestoquard (F.), Plantureux (Ed.) et Rovgebief (H.). - Les piroplasmoses bovines d'Algérie. Arch. Inst. Pasteur Algérie, II, 1924, p. 1-147.

Wenyon (C.-M.). - Protozoology. London, 1926.

Laboraloire de Parasilologie de Stanleyville. 